\title{
An unusual case of mediastinal mass due to Fonsecaea pedrosoi
}

\author{
N. Singh*, R. Agarwal*, D. Gupta*, M.R. Shivaprakash" and A. Chakrabarti ${ }^{\#}$
}

\begin{abstract}
Phaeohyphomycosis is a disease caused by dematiaceous fungi. Here, the first culture-proven case of mediastinal mass due to Fonsecaea pedrosoi is reported.

The patient was a 40 -yr-old male who had presented with history of dyspnoea and dysphagia.

Computed tomography of the chest disclosed a mediastinal mass. Cultures of a specimen from the mediastinum grew Fonsecaea pedrosoi. The patient received amphotericin $B$ and itraconazole and showed a remarkable recovery. Repeat computed tomography revealed a reduction in the mediastinal mass.
\end{abstract}

The case highlights the need for a high degree of clinical suspicion and appropriate histopathological and mycological examination of clinical specimens.

\section{KEYWORDS: Fonsecaea pedrosoi, itraconazole, mediastinal mass}

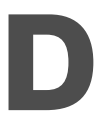
ematiaceous fungi are the aetiological agents of phaeohyphomycosis, and are now increasingly being recognised as causing disease in humans. Diverse presentations of infectious syndromes are seen, ranging from local infections due to trauma to widely disseminated infection in immunocompromised patients. Pulmonary disease generally includes allergic bronchopulmonary mycosis, pulmonary nodules and endobronchial lesions. A high level of suspicion and routine fungal cultures are required to identify these cases. There is no consensus regarding the management of these cases. Itraconazole has the most consistent in vitro activity compared to other antifungal drugs. Further studies are needed to better understand the pathogenesis and treatment of these uncommon infections. Here, an unusual presentation of phaeohyphomycosis (secondary to Fonsecaea pedrosoi) presenting as a mediastinal mass is described.

\section{CASE REPORT}

A 38-yr-old, apparently immunocompetent, male presented with a history of dysphagia of 6 months' duration. The dysphagia had appeared insidiously, was present for both solids and liquids, and had slowly progressed to absolute dysphagia at the time of presentation. For 2 weeks, the dysphagia had been accompanied by hoarseness of voice and nonproductive cough. The patient was investigated at another centre, where a computed tomographic scan of his chest revealed the presence of a large lobulated mass lesion of heterogeneous intensity extending up to the level of the carina (fig. 1). An ultrasoundguided Trucut biopsy specimen from the mediastinum revealed multiple granulomas and giant cells along with fibrosis. The patient was started on antitubercular drugs $\left(5 \mathrm{mg} \cdot \mathrm{kg}^{-1}\right.$ isoniazid, $10 \mathrm{mg} \cdot \mathrm{kg}^{-1}$ rifampicin, $25 \mathrm{mg} \cdot \mathrm{kg}^{-1}$ pyrazinamide and $15 \mathrm{mg} \cdot \mathrm{kg}^{-1}$ ethambutol) and $20 \mathrm{mg} \cdot \mathrm{day}^{-1}$ prednisolone. He did not show any improvement and his symptoms worsened. Rapid-onset dyspnoea and absolute dysphagia had necessitated tracheostomy and feeding jejunostomy at the referring institute prior to admission to the Post Graduate Institute of Medical Education and Research (Chandigarh, India).

On examination at the Post Graduate Institute of Medical Education and Research, the patient was conscious and obeying commands. $\mathrm{He}$ was afebrile and his vital signs were stable (pulse rate 92 beats $\mathrm{min}^{-1}$, blood pressure $110 / 80 \mathrm{mmHg}$ and respiratory frequency 18 breaths $\cdot \mathrm{min}^{-1}$ ). Examination of the abdomen, nervous system, and cardiovascular and respiratory systems revealed that they were within normal limits. Laboratory investigations revealed mild normocytic normochromic anaemia and hypoalbuminaemia. A repeat histopathological examination of the specimens obtained from the mediastinum at the Post Graduate Institute of Medical Education and Research showed numerous granulomas with associated mononuclear cell

\section{AFFLLIATIONS}

Depts of *Pulmonary Medicine, and

" Medical Microbiology, Post

Graduate Institute of Medical

Education and Research, Chandigarh, India.

CORRESPONDENCE

N. Singh

Dept of Pulmonary Medicine

Post Graduate Institute of Medical

Education and Research

Sector 12

Chandigarh

India 160012

Fax: 911722748215

E-mail: navneetchd@yahoo.com

Received:

December 082005

Accepted after revision:

February 172006 

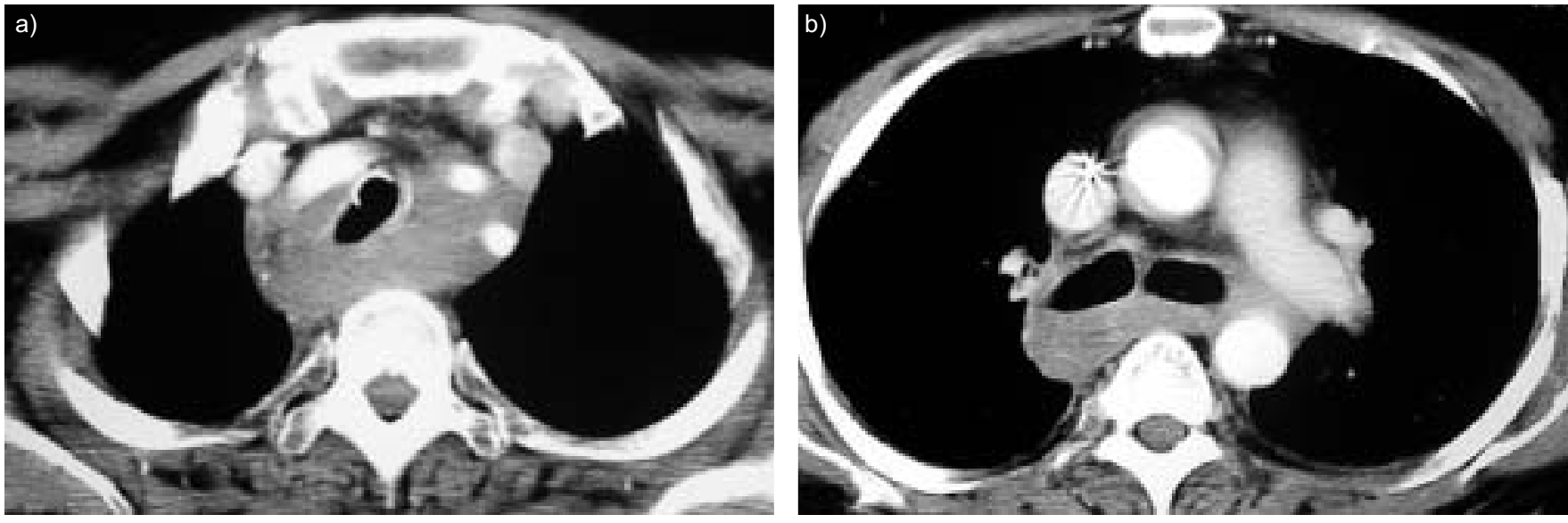

FIGURE 1. Computed tomographic scan of the chest showing a soft-tissue density swelling in the mediastinum just below the thoracic inlet (a) and extending up to the level of the carina (b).

infiltrate; septate hyphae within the areas of inflammation were also identified. Fungal serology performed for Candida and Aspergillus was negative. Blood and urine cultures did not reveal the growth of any fungus. Results of radiography and a computed tomographic scan of the paranasal sinuses were normal. Serology for HIV was nonreactive, and the fasting plasma glucose level was within normal limits.

With a provisional diagnosis of mediastinal mass secondary to a fungal infection (provisionally Aspergillus), the patient was started on intravenous amphotericin B, but showed little improvement. With the intention of obtaining material for the identification of the causative fungus, ultrasound-guided fineneedle aspiration from the mediastinal mass was carried out. It revealed few multinucleate giant cells, stromal fragments and focal necrosis, along with similar fungal profiles as seen on previous biopsy. However, the growth on Sabouraud dextrose agar did not conform to that of Aspergillus, and, on further characterisation, including staining (lactophenol cotton blue mount), F. pedrosoi was identified (fig. 2). Oral itraconazole

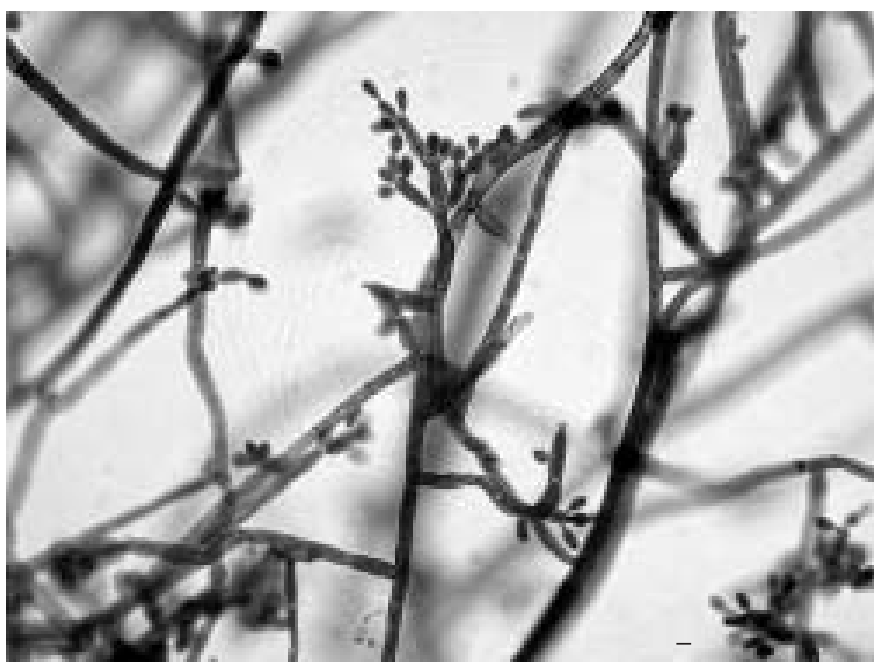

FIGURE 2. Photomicrograph showing short chains and round spores of Fonsecaea pedrosoi (lactophenol cotton blue mount). Scale bar $=1 \mu \mathrm{m}$.
$200 \mathrm{mg} \cdot$ day $^{-1}$ was added to the amphotericin B; amphotericin B was stopped after a cumulative dose of $2 \mathrm{~g}$.

The patient showed gradual improvement and was discharged on oral itraconazole. The patient continues on maintenance therapy with oral itraconazole and has shown subjective improvement in the form of regression of all symptoms. His jejunostomy opening has been closed and decannulation of the tracheostomy is continuing. Repeat computed tomography of the chest performed after completion of 6 months of itraconazole therapy showed a reduction in the size of the mediastinal mass (fig. 3).

\section{DISCUSSION}

Dematiaceous fungi, including F. pedrosoi, are a group of heterogeneous ubiquitous fungi known to cause phaeohyphomycosis, a spectrum of disease ranging from superficial to deep-seated infections [1]. In contrast to superficial involvement (cutaneous and subcutaneous disease), deep-seated disease is far less common. The distinguishing characteristic common to these fungi is the presence of melanin in their cell wall, which is also believed to be a virulence factor.

The commonest presentation of systemic phaeohyphomycosis is in the form of cerebral involvement (such as a brain abscess), other localised infections (such as pneumonia, pulmonary nodules and endobronchial lesions), and disseminated infections. Neurological involvement alone can occur in immunocompetent individuals without any obvious risk factors [2], in contrast to the involvement of other sites or the disseminated disease that tends to occur mainly in the setting of immunosuppression [3]. The most commonly involved genera are Scedosporium (disseminated), Cladophialophora (neurological) and Ochroconis (pulmonary) [2-4]. The genus Fonsecaea is a rare cause of phaeohyphomycosis; only one out of 72 cases was reported in a series of disseminated disease, and three out of 101 in primary central nervous system phaeohyphomycosis. To the best of the present authors' knowledge, this is the first report of pulmonary involvement by Fonsecaea, and also the first case report of a mediastinal mass due to dematiaceous fungi. 

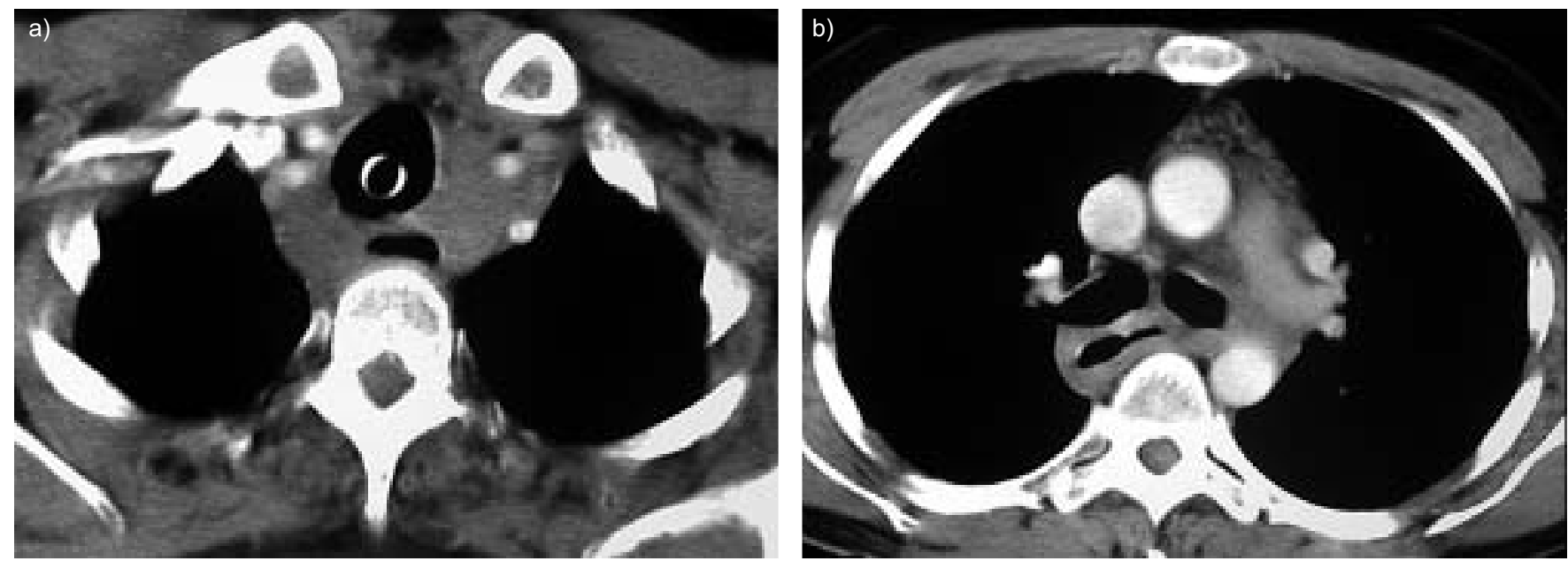

FIGURE 3. Computed tomography scan of the chest showing a marked reduction in the soft-tissue density swelling just below the thoracic inlet (a) and extending up to the level of the carina ( $b$; oesophageal opening not visible in figure 1 can be seen here).

Systemic phaeohyphomycosis is difficult to treat and carries a dismal prognosis, one of the reasons being the relative lack of response to amphotericin B. Recovery is usually seen in patients in whom combined medical and surgical management has been aggressively and timely initiated [2]. There is no standardised therapy, and combination of amphotericin B with other antimycotic agents, such as itraconazole, may improve survival rates. In a study of the in vitro susceptibility of 12 primary human isolates of $F$. pedrosoi, resistance to amphotericin B, 5-flucytosine and fluconazole was seen in 33.0, 58.3 and $66.7 \%$, respectively, in contrast to $0 \%$ for itraconazole [5]. In another series by SHARKEY et al. [6], in 17 patients with phaeohyphomycosis involving different organs (including five in whom amphotericin B therapy alone and five in whom combination amphotericin B and imidazole therapy had failed), itraconazole in doses ranging 50-600 mg day ${ }^{-1}$, when used for 1-48 months, led to clinical improvement or remission in nine patients and stabilisation of disease in two, prompting the authors to conclude that itraconazole could be used as the first line of drug therapy for this mycosis [6, 7]. Voriconazole might have a role in therapy, but there is no clinical experience to date.

The case presented had certain unusual features. The disease was caused by an unusual fungus (Fonsecaea), occurred at an unusual site (mediastinum) and in an immunocompetent individual, and responded to medical therapy alone. Indeed, long-term treatment with itraconazole led to improvement even when the patient had not responded to therapy with amphotericin B.

In conclusion, a high index of suspicion in both treating clinicians and microbiologists is necessary for the diagnosis of this entity, the reasons being the rarity of the disorder and the need for prolonged treatment, once identified. Also, in areas of high prevalence of Mycobacterium tuberculosis, the identification of granulomas histopathologically could lead to inadvertent treatment with antitubercular drugs, as seen in the present patient. In every case in which fungal infection is suspected, attempts must always be made to identify the specific fungus using appropriate microbiological and histopathological methods and not to stop investigations after mere documentation of fungal profiles on staining. Confirmation of the specific pathogen (such as Fonsecaea pedrosoi, in this case) would lead to not only initiation of the proper therapy but also prevention of premature discontinuation of therapy that would potentially deny the benefit of getting cured to the rare patient who may be unfortunate enough to suffer from this disease.

\section{REFERENCES}

1 Brandt ME, Warnock DW. Epidemiology, clinical manifestations and therapy of infections caused by dematiaceous fungi. J Chemother 2003; 15: Suppl. 2, 36-47.

2 Revankar SG, Sutton DA, Rinaldi MG. Primary central nervous system phaeohyphomycosis: a review of 101 cases. Clin Infect Dis 2004; 38: 206-216.

3 Revankar SG, Patterson JE, Sutton DA, Pullen R, Rinaldi MG. Disseminated phaeohyphomycosis: review of an emerging mycosis. Clin Infect Dis 2002; 34: 467-476.

4 Revankar SG. Dematiaceous fungi. Semin Respir Crit Care Med 2004; 25: 183-189.

5 de Bedout C, Gomez BL, Restrepo A. In vitro susceptibility testing of Fonsecaea pedrosoi to antifungals. Rev Inst Med Trop Sao Paulo 1997; 39: 145-148.

6 Sharkey PK, Graybill JR, Rinaldi MG, et al. Itraconazole treatment of phaeohyphomycosis. J Am Acad Dermatol 1990; 23: 577-586.

7 Rinaldi MG. Phaeohyphomycosis. Dermatol Clin 1996; 14: 147-153. 